\title{
Evolutionary and sequence-based relationships in bacterial AdoMet-dependent non-coding RNA methyltransferases
}

\author{
Jeanneth Mosquera-Rendón ${ }^{1,2}$, Sonia Cárdenas-Brito ${ }^{1}$, Juan D Pineda ${ }^{3}$, Mauricio Corredor ${ }^{1,2}$
}

and Alfonso Benítez-Páez ${ }^{1 *}$

\begin{abstract}
Background: RNA post-transcriptional modification is an exciting field of research that has evidenced this editing process as a sophisticated epigenetic mechanism to fine tune the ribosome function and to control gene expression. Although tRNA modifications seem to be more relevant for the ribosome function and cell physiology as a whole, some rRNA modifications have also been seen to play pivotal roles, essentially those located in central ribosome regions. RNA methylation at nucleobases and ribose moieties of nucleotides appear to frequently modulate its chemistry and structure. RNA methyltransferases comprise a superfamily of highly specialized enzymes that accomplish a wide variety of modifications. These enzymes exhibit a poor degree of sequence similarity in spite of using a common reaction cofactor and modifying the same substrate type.

Results: Relationships and lineages of RNA methyltransferases have been extensively discussed, but no consensus has been reached. To shed light on this topic, we performed amino acid and codon-based sequence analyses to determine phylogenetic relationships and molecular evolution. We found that most Class I RNA MTases are evolutionarily related to protein and cofactor/vitamin biosynthesis methyltransferases. Additionally, we found that at least nine lineages explain the diversity of RNA MTases. We evidenced that RNA methyltransferases have high content of polar and positively charged amino acid, which coincides with the electrochemistry of their substrates.

Conclusions: After studying almost 12,000 bacterial genomes and 2,000 patho-pangenomes, we revealed that molecular evolution of Class I methyltransferases matches the different rates of synonymous and non-synonymous substitutions along the coding region. Consequently, evolution on Class I methyltransferases selects against amino acid changes affecting the structure conformation.
\end{abstract}

Keywords: Molecular evolution, RNA methyltransferases, Bacteria, Conserved sequence motifs, Antibiotic resistance

\section{Background}

Post-transcriptional modifications of nucleotides in RNA molecules, such as ribosome and transfer RNA (rRNA and tRNA, respectively), is a process observed in the three major kingdoms of life: Archea, Eukarya and Bacteria. It is evidenced as a sophisticated epigenetic mechanism involved in translation accuracy and gene expression control. RNA modifications appear to confer structural stability $[1,2]$ and to participate in translation fidelity $[3,4]$.

\footnotetext{
*Correspondence: abenitez@cidbio.org

'Bioinformatics Analysis Group - GABi, Centro de Investigación y Desarrollo en Biotecnología - CIDBIO, 111221 Bogotá, D.C, Colombia

Full list of author information is available at the end of the article
}

Among the wide variety of modifications found in rRNAs and tRNAs, uridine isomerization (pseudourdine synthesis) and the methylation of nucleobases and/or ribose moieties of nucleotides are predominantly present in these central biomolecules [5,6]. Bacterial RNA modification is enzyme-dependent, thus involving a broad variety of protein families that are highly specialized in both the reaction and substrate to be modified $[5,6]$. Notwithstanding, very recent reports have demonstrated dual specificity and activity [7-10]. One interesting group of proteins acting as RNA-modifying enzymes is composed of AdoMet(or S-adenosyl-L-methionine) dependent RNA methyltransferases (MTases). Globally, enzymes that methylate 
RNA comprise two major classes of MTases according to their structure core: i) Rossmann-Fold MTases (RFM) including almost all the $\mathrm{N}$ and $\mathrm{C}$ methylases and modify nucleobases; ii) SPOUT MTases consisting of 2'$O$-methylases which act essentially in tRNAs with very few exceptions $[11,12]$. However, a later classification of MTases distinguishes five structurally different classes of MTases denoted as I (RFM), II, III, IV (SPOUT) and $\mathrm{V}$ [13]. Interestingly, the global sequence conservation among all the MTases classes is poor, which hinders the proposal of phylogenetic relationships. However, they structurally manifest an analogous architecture as a result of using AdoMet as a cofactor of the methyltransfer reaction $[13,14]$. Class I MTases comprise most rRNAmodifying enzymes (and DNA MTases) showing a fair degree of sequence similarity [6]. The low degree of sequence similarity observed in the predominantly Class I MTases hinders study of their evolutionary history. Although an extensive duplication and specialization process in evolution is thought to produce multiple families of known RNA MTases, the possibility that multiple lineages of RNA MTases can emerge cannot be ruled out $[12,13]$. Most rRNA MTases are well-conserved only in bacteria and cannot be traced in other kingdoms such as Eukarya. Nevertheless, a few genes (i.e., $\operatorname{rsm} A$ ) display a wide phylogenetic distribution that confers these conserved MTases a relevant role in decoding both the function and biogenesis of the ribosome $[12,15,16]$. Certain indigenous RNA methylations have been characterized as being pivotal to maintain ribosome fidelity [7,17-20], and one of them has even been characterized as indispensable for cell growth, indicating a critical role for the proper ribosome function [21]. Alternatively, the mutations at ribosome genes, such as rRNA MTases, appear to be frequently associated with conferring antibiotic resistance. One of the first reported cases is $\operatorname{rsm} A$ mutation inactivating the RsmA function and promoting Kasugamycin resistance [22-24]. Another well-known case of antibiotic resistance associated with mutations in rRNA MTases is the rsmG gene [25-27]. The global mechanisms of the initial state of low-level resistance and the later acquisition of high-level resistance seems similar among strains and genes [27,28]. All the above-mentioned effects of RNA methylation deficiency on cell physiology, as well as the well-known antibiotic resistance phenomena by plasmid-encoded RNA MTases [29-33], are thought to design new antimicrobial strategies.

With the recent characterization of YhiR as the RlmJ MTase that acts on 23S rRNA from Escherichia coli [34], the full set of RNA MTases for this model organism have been depicted (see Table 1). Currently, research aims are conducted to disclose both the RNA modifications and responsible enzymes in other model organisms as part of the Modomics field of RNA biology. Notwithstanding, further sequence, structural, and functional characterizations of the known RNA MTases are absolutely essential to: i) clarify the critical amino acids for the function and specificity of MTases; ii) disclose potential new catalytic mechanisms; iii) study the structural rearrangements that some MTases undergo to perform their functions; iv) acquire knowledge of the dual activities of RNA MTases, which are becoming a more frequent event than expected; and v) shed light on the evolutionary origin and relationships among RNA MTases. In recent years, several threedimensional structures have been solved and some offer insights into catalytic mechanisms of nucleotide methylation [8,35-37]. Similarly, relevant genomic studies have presented important phylogenetic and evolutionary features of RNA MTases [11,38]. Moreover, with the full set of known RNA MTases characterized for the model organism Escherichia coli, new large-scale sequence and genomic studies into the function, variation and diversity of these enzymes responsible for RNA methylation can lead to a better understanding of the origin of this superfamily of enzymes and shed light on both their evolutionarily meaning as well as the link between RNA methylations and bacterial antibiotic resistance.

\section{Results and discussion}

\section{Evolutionary conservation of RNA MTases}

After collecting the full set of RNA MTases acting in both rRNAs and tRNAs from Escherichia coli (see Table 1), we recovered homologs for each family of RNA MTases by using this set of proteins as a query in a Blastp-based searching. Thus, we recovered almost 3,000 different sequences, which represent a high level of diversity for these MTases in Eubacteria. We built a UPGMA-based dendrogram using the phylogenetic information obtained from RNA MTases across bacterial species (Figure 1). This dendrogram reflects relationships among RNA MTase families according to their distribution in major bacteria phyla. Globally, two major groups of MTases are observed, considered to be those enzymes with a mid to low conservation across species and those that are very well-conserved. In this latter group, a core of enzymes required for the proper ribosome function is distinguished. Consequently, $16 \mathrm{~S}$ rRNA MTases RsmG, RsmH/I, and RsmE emerge as the highly conserved accessory proteins of the prokaryote ribosome, and their relevance for translation is further supported by the fact that their products $\mathrm{m}^{7} \mathrm{G} 527, \mathrm{~m}^{4} \mathrm{Cm} 1402$ and $\mathrm{m}^{3} \mathrm{U} 1498$, respectively, lie on rRNA regions and play pivotal roles in the decoding function [70,71]. Likewise, the high evolutionary conservation of RsmB (responsible for the $\mathrm{m}^{5} \mathrm{C} 967$ modification) matches an important role of its target in the ribosome function [72]. Regarding the evolutionary conserved pattern of $23 \mathrm{~S}$ rRNA MTases, we observed that $R \operatorname{lm} H, R \operatorname{lmB}$ and $R \operatorname{lmN}$, 
Table 1 Set of the $E$. coli RNA MTases used as bait in this study

\begin{tabular}{|c|c|c|c|c|c|c|}
\hline Name & Alternative Name(s) & Substrate & Modification & MTase Family ${ }^{\mathbf{A}}$ & Uniprot & Reference \\
\hline $\mathrm{R} / \mathrm{mA}$ & RrmA, YebH & $23 \mathrm{~S}$ rRNA & $m^{1} G 745$ & Class I (RFM) & P36999 & [39] \\
\hline RlmB & YfjH & $23 \mathrm{~S}$ rRNA & Gm2251 & Class IV (SPOUT) & P63177 & {$[40]$} \\
\hline RImC & RumB, YbjF & $23 \mathrm{~S}$ rRNA & $m^{5} U 747$ & Class I (RFM) & P75817 & [41] \\
\hline RImD & RumA, YgcA & $23 \mathrm{~S}$ rRNA & $m^{5} U 1939$ & Class I (RMF) & P55135 & {$[42]$} \\
\hline RImE & FtsJ, MrsF, RrmJ & $23 \mathrm{~S}$ rRNA & Um2552 & Class IV (SPOUT) & POCOR7 & [43] \\
\hline $\mathrm{RImF}$ & YbiN & $23 \mathrm{~S}$ rRNA & $m^{6} \mathrm{~A} 1618$ & Class I (RFM) & P75782 & {$[44]$} \\
\hline RImG & YgjO & $23 \mathrm{~S}$ rRNA & $m^{2} \mathrm{G} 1835$ & Class I (RFM) & P42596 & {$[45]$} \\
\hline $\mathrm{RImH}$ & YbeA & $23 \mathrm{~S}$ rRNA & $m^{3} \psi 1915$ & Class IV (SPOUT) & POA8I8 & {$[44]$} \\
\hline Rlml & YccW & $23 \mathrm{~S}$ rRNA & $m^{5} \mathrm{C} 1962$ & Class I (RFM) & P75876 & {$[46]$} \\
\hline $\mathrm{R} / \mathrm{m} J$ & YhiR & $23 \mathrm{~S}$ rRNA & $m^{6}$ A2030 & Class I (RFM) & P37634 & {$[34]$} \\
\hline $\operatorname{RImK} / L^{*}$ & YcbY & $23 \mathrm{~S}$ rRNA & $m^{7} G 2069 / m^{2} G 2445$ & Class I (RFM) & P75864 & {$[47-49]$} \\
\hline RImM & YgdE & $23 \mathrm{~S}$ rRNA & Cm2498 & Class I (RFM) & POADR6 & {$[50]$} \\
\hline $\mathrm{RImN}$ & $Y f g B$ & $23 \mathrm{~S}$ rRNA / tRNAs & m2A2503 / m2A37 & Radical SAM & P36979 & {$[7,51]$} \\
\hline RsmA & KsgA & $16 \mathrm{~S}$ rRNA & $m_{2}^{6} A 1518 / m_{2}^{6} A 1519$ & Class I (RFM) & P06992 & {$[52]$} \\
\hline RsmB & RrmB, YhdB, Sun & $16 \mathrm{~S}$ rRNA & $m^{5} \mathrm{C} 967$ & Class I (RFM) & P36929 & {$[53,54]$} \\
\hline RsmC & YjjT & $16 \mathrm{~S}$ rRNA & $m^{2} \mathrm{G} 1207$ & Class I (RFM) & P39406 & {$[55]$} \\
\hline RsmD & YhhF & $16 \mathrm{~S}$ rRNA & $m^{2} \mathrm{G} 966$ & Class I (RFM) & POADX9 & [56] \\
\hline RsmE & Ygg」 & $16 \mathrm{~S}$ rRNA & $m^{3} \mathrm{U} 1498$ & Class IV (SPOUT) & POAGL7 & {$[57]$} \\
\hline RsmF & YebU & $16 \mathrm{~S}$ rRNA & $m^{5} \mathrm{C} 1407$ & Class I (RFM) & P76273 & {$[58]$} \\
\hline RsmG & GidB & $16 \mathrm{~S}$ rRNA & $m^{7} G 527$ & Class I (RFM) & P0A6U5 & [27] \\
\hline $\mathrm{RsmH}$ & MraW, YabC & $16 \mathrm{~S}$ rRNA & $m^{4} \mathrm{C} 1402$ & Class I (RFM) & P60390 & {$[18]$} \\
\hline Rsml & YraL & $16 \mathrm{~S}$ rRNA & Cm1402 & Class I (RFM) & P67087 & [18] \\
\hline RsmJ & YhiQ & $16 \mathrm{~S}$ rRNA & $m^{2} \mathrm{G} 1516$ & Class I (RFM) & P68567 & [59] \\
\hline TrmA & RumT & tRNAs / tmRNAs & $m^{5} \cup 54 / m^{5} \cup 341$ & Class I (RFM) & P23003 & {$[9,60]$} \\
\hline $\operatorname{TrmB}$ & $\mathrm{YggH}$ & tRNAs & $m^{7} G 46$ & Class I (RFM) & P0A815 & {$[61]$} \\
\hline TrmD & TrmD & tRNAs & $m^{1} \mathrm{G} 37$ & Class IV (SPOUT) & P0A873 & {$[62]$} \\
\hline TrmH & SpoU & tRNAs & Gm18 & Class IV (SPOUT) & POAGJ2 & {$[63]$} \\
\hline Trm」 & YfhQ & tRNAs & Um32 / Cm32 & Class IV (SPOUT) & POAE01 & {$[64]$} \\
\hline TrmL & YibK & tRNAs & Um34 / Cm34 & Class IV (SPOUT) & P0AGJ7 & {$[65]$} \\
\hline TrmN6 & YfiC & tRNAs & $\mathrm{m}^{6} \mathrm{~A} 37$ & Class I (RFM) & P31825 & {$[66]$} \\
\hline$M n m C^{B}$ & YfcK, TrmC & tRNAs & $m n m^{5} U$ & Class I (RFM) & P77182 & {$[67,68]$} \\
\hline $\mathrm{CmoA}$ & $\mathrm{YecO}$ & tRNAs & $\mathrm{cmo}^{5} \mathrm{U}$ & Class I (RFM) & P76290 & [69] \\
\hline $\mathrm{CmoB}$ & YecP & tRNAs & $\mathrm{cmo}^{5} \mathrm{U}$ & Class I (RFM) & P76291 & {$[69]$} \\
\hline
\end{tabular}

*We split this fused-domain MTase to assume independent MTases during the homolog search. A) Classification according to amino acid motifs and published structure when available; RFM = Rossmann Fold Methyltransferase; SPOUT=Similar to the SpoU MTase structure. B) Only refers to the N-terminal MTase domain of the bi-functional MnmC protein.

producing $\mathrm{m}^{3} \Psi 1915$, Gm2251 and $\mathrm{m}^{2} \mathrm{~A} 2503$, respectively, are present in most bacterial species with very few exceptions. Similarly to the conserved $16 \mathrm{~S}$ rRNA MTases, these three enzymes act on central sites for the ribosome function located close to the Petidyl Transferase Center (PTC). The pivotal role of these modifications is well supported given that inactivation of respective 23S rRNA MTases function has been shown to have negative effects on translation and cell physiology [7,51,73,74]. Interestingly, almost all of these sites and/or regions of $16 \mathrm{~S}$ and $23 \mathrm{~S}$
rRNA showing a conserved methylation pattern appear to be associated with antibiotic resistance. As a consequence, alteration of methylation patterns on rRNA has been associated aminoglycoside [24,27], tetracycline [75], tylosin [76], linezolid [77,78], and chloramphenicol resistance [79] as well as PhLOPSA multiresistance [80]. All this evidence could indicate that rRNA methylation emerges as a new molecular mechanism mediating bacterial resistance. This last issue is intriguing given that mutations in RNA MTases often produce associated fitness cost $[18,44,51]$. 


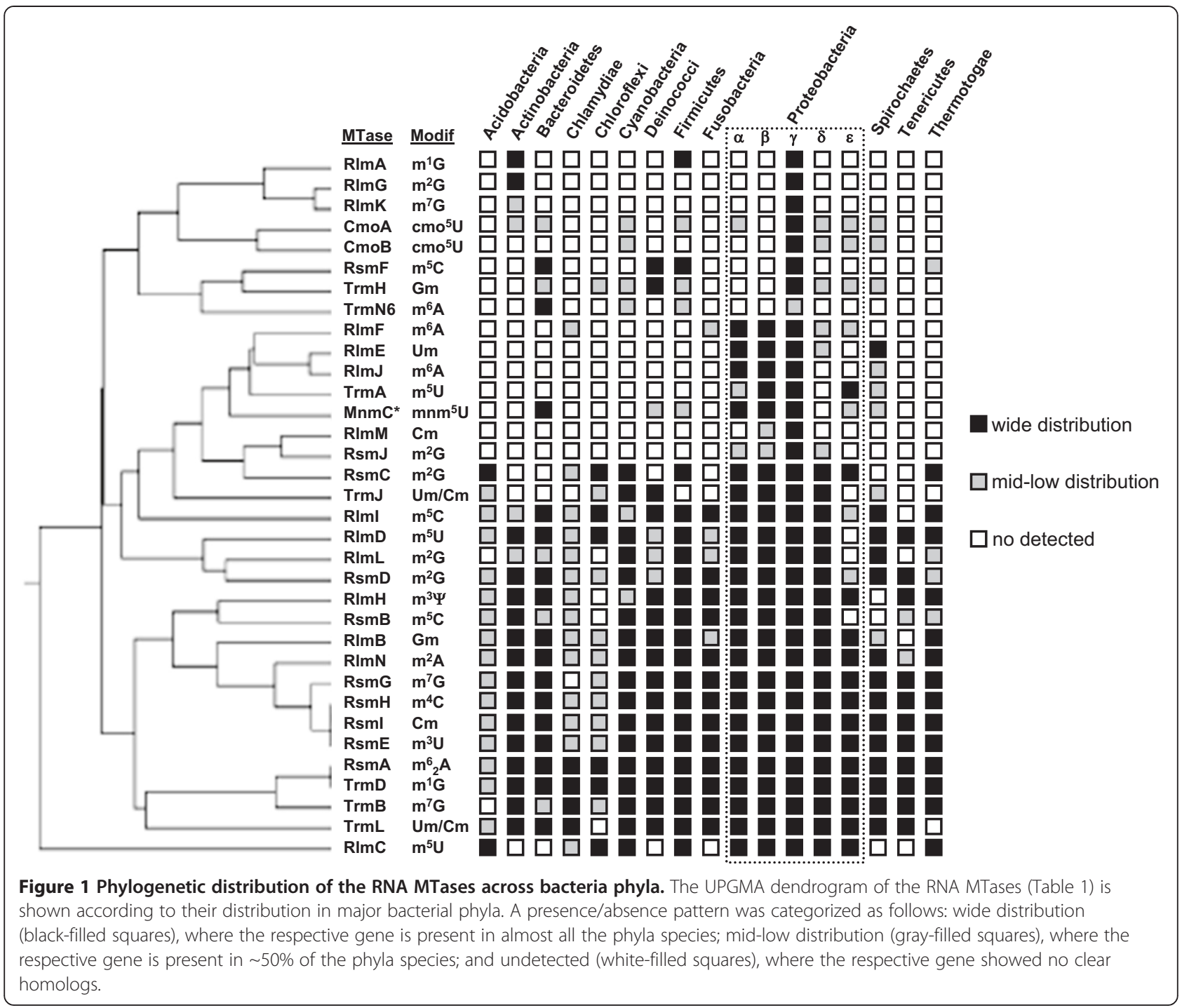

Consequently, the process of antibiotic resistance acquisition, normally initiated with low-level resistances, requires further study in order to disclose the genetic and physiology basis of this short-term evolutionary process. Regarding the RNA MTases acting on tRNAs, the TrmD, TrmB, and TrmL MTases also appear to be highly conserved among bacteria. TrmD and TrmL are responsible for the pivotal modifications occurring in the anticodon region of tRNAs, where they are directly involved in proper mRNA decoding $[19,65]$. In global terms, approximately half the studied enzymes can constitute the minimal set of methylations at rRNA and tRNAs required for life. This estimation slightly differs when allelic genes encoding enzymes responsible of universally conserved modifications like $\mathrm{m}^{5} \mathrm{U} 54$ are considered [81]. In a similar manner, we hypothesize that the modification performed by the poorly conserved RsmF protein can be made by other enzymes because several paralogs of this protein have been detected (see Figure 2). Strikingly, the RNA MTases responsible for $\mathrm{m}^{6} \mathrm{~A}$ modifications seem to display low conservation (except the RsmA dimethylase enzyme). This fact could indicate that acquisition of this modification type is a recent event during evolution.

Sequence-based relationships among RNA MTases (the similarity network)

In addition to presenting the phylogenetic occurrence of RNA MTases across bacterial species, we analyzed the sequence-based relationships among different MTase families to trace their evolutionary origin. With the aim to shed light on this topic, we performed an extensive sequence analysis using probabilistic inference methods (HMMER3 based analysis), a distant homolog searching algorithm (PSI-Coffee analysis) and the information retrieved from almost 3,000 amino acid sequences. Consequently, the respective amino acid profiles obtained for 


\section{S rRNA MTases}
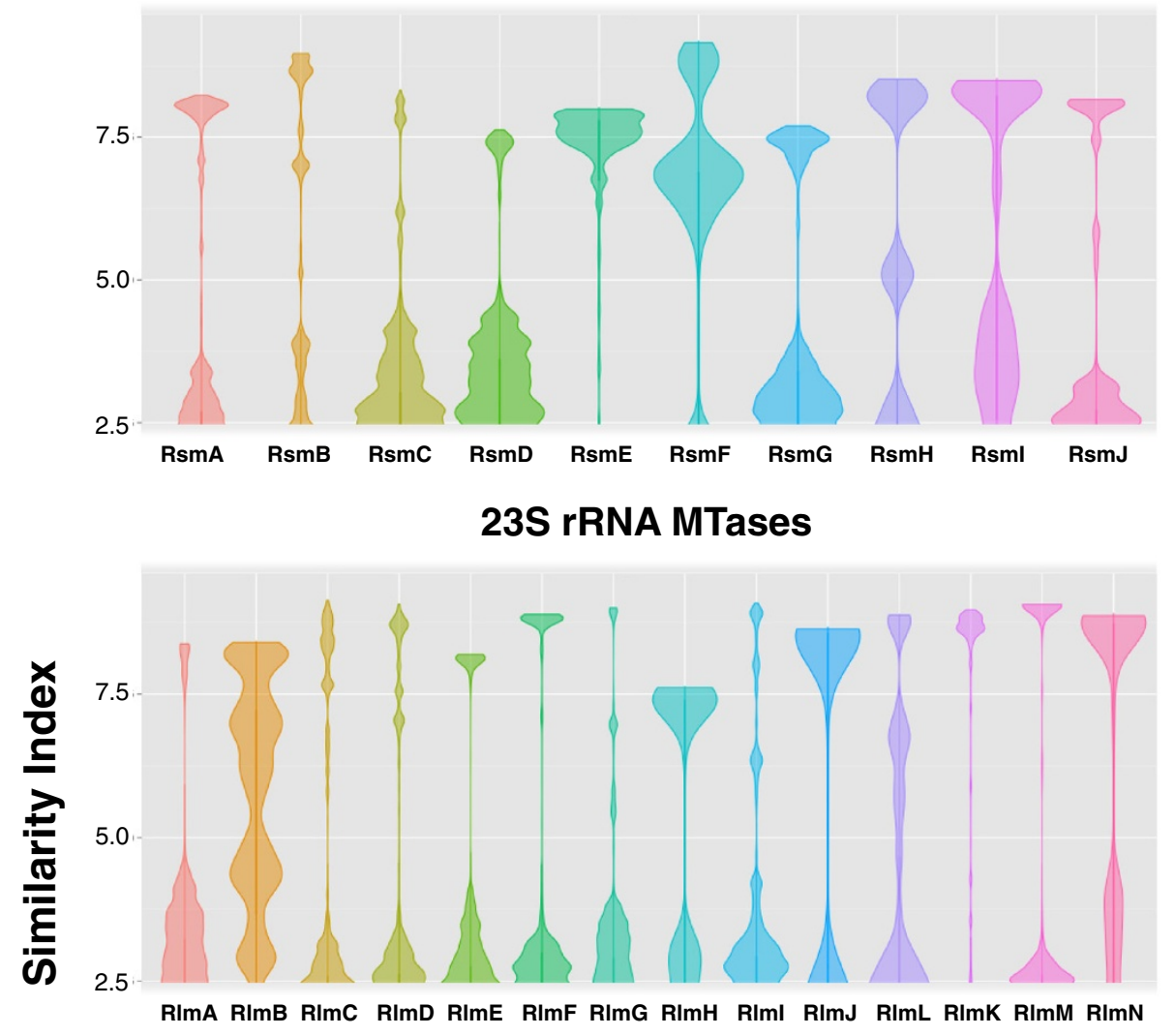

tRNA MTases

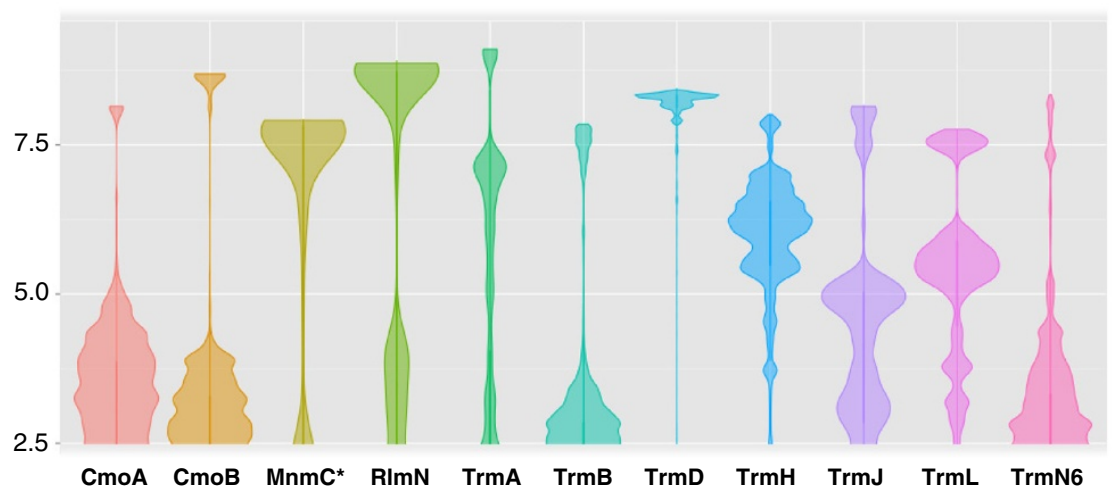

Figure 2 Distribution of the RNA MTase sequence motifs across bacterial genomes. Using the amino acid profiles inferred from the probabilistic methods, a search for the proteins matching the RNA MTase sequences was done. Information on query and alignment length, and on the score for amino acid replacements was used to draw the density violin plots per family and class of RNA MTases. Orthology was considered for those hits (with few exceptions) with a Similarity Index higher than 7.5, whereas paralogy was considered for hits with a Similarity Index 5.0 to 7.5 . * Refers to the N-terminal domain of the bi-functional MnmC enzyme.

each family of RNA MTases described in E. coli (see Table 1) were used to rescue proteins with similar amino acid patterns along the Escherichia coli K12 genome and other genomes from model organisms such as Bacillus subtilis 168. We represented the sequence matches among families (nodes) in a network fashion by scoring the interactions (edges) with a Similarity Index calculated from different alignment parameters such as length and amino acid substitutions (see Amino acid profiles at Methods). Similarity index higher than 2.5 supported trusted relationships between proteins, this is, sequence alignments at least 35 amino acids in length ( $15 \%$ of the average size of MTases). Figure 3A illustrates the network representing the relationships among the different MTases in E. coli. Accordingly, 
we found RNA MTases have several sequence patterns present in other AdoMet-dependent MTases, including the Ribosome Protein Methyltransferases (orange nodes) and the MTases involved in the biosynthesis of cofactors/ vitamins (gray nodes). Strikingly, no sequence-based relationships were detected with DNA MTases; these relationships would be expected given the similar nature of the substrates on which both types of MTases act. Consequently, these results indicate that the sequence similarities observed in our analysis are based on certain relationships with no bias by substrate preference. The network also represents three major lineages of MTases that are reproducible in E. coli and B. subtilis (Figure 3C), most of which are Class I MTases (a big cluster of nodes), SPOUT MTases (the gray-shaded cluster), and the RsmB/F cluster (the blue-shaded cluster). This last group of proteins was clearly separated from the other Class I MTases in $B$. subtilis (Figure 3C). The clustering of the well-defined SPOUT [11,38] and RsmB/F [58] lineages also supports our results and reinforces the idea of a single lineage comprised of the majority of the Class I MTases acting in different types of substrates. After performing a cluster analysis based on edges scores and different interactions (see Amino acid profiles at Methods), the Multifunction Cluster of MTases in both model organisms was split into two sub-populations. Although no clear distribution of functions was seen, one of the groups was predominantly made up of RNA and Protein MTases, whereas the other one was constituted predominantly by the MTases involved in cofactor/vitamin biosynthesis and unknown function MTases, which could well be predicted for this molecular function.

\section{Origin and lineages of RNA MTases}

Interestingly, a special group of MTases was always present in the transition between both the sub-populations of the Multifunction Cluster of MTases, where greater connectivity was present. Figures 3B and 3D show such

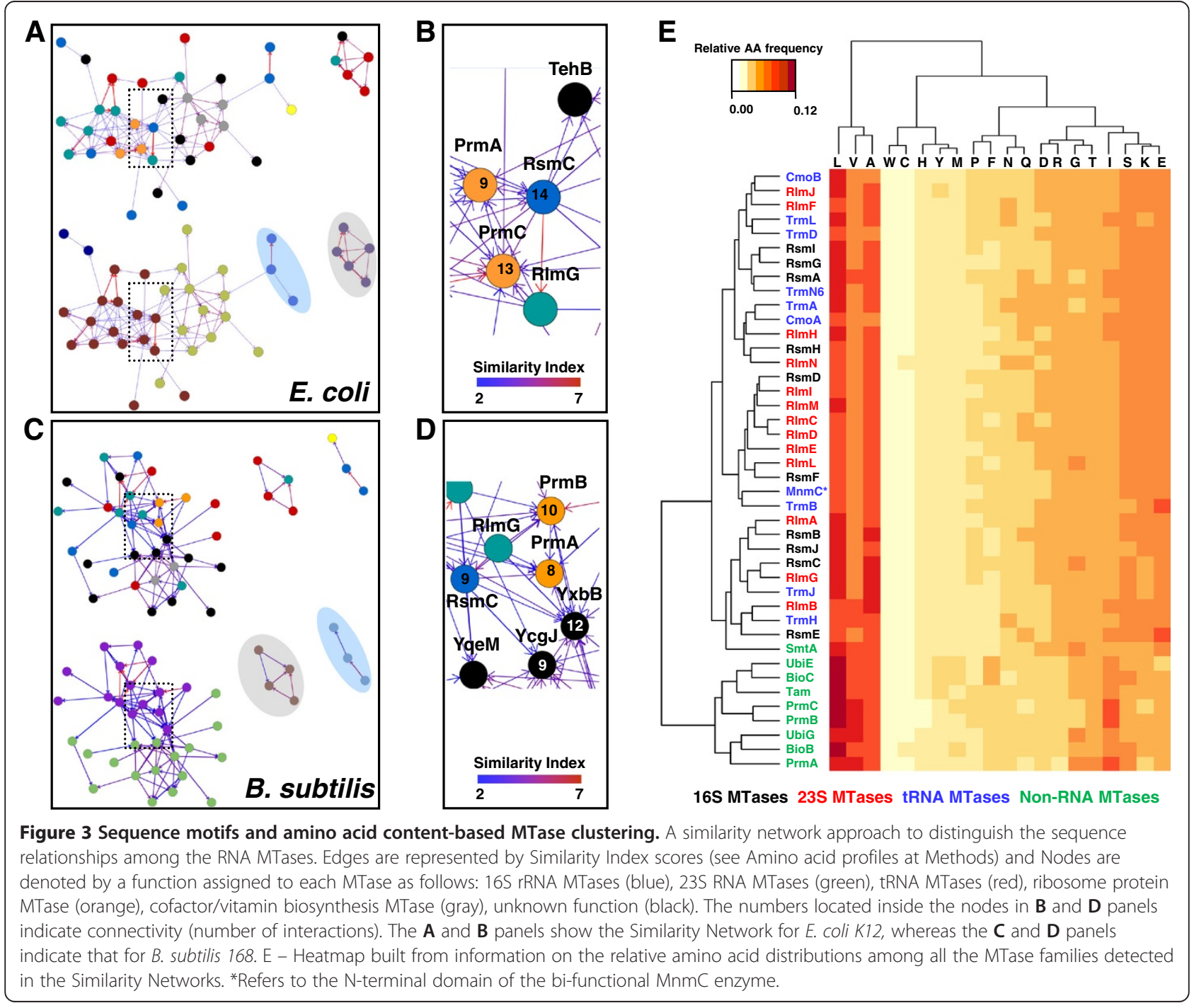


relevant nodes in the network. Thus, the RsmC, PrmA, PrmB, and PrmC proteins obtained the higher connectivity values in respective networks, indicating that one of these families could be the original member of this Multifunction Cluster of MTases. These results were similarly found in the MTase networks built for Mycobacterium tuberculosis H37Rv, Pseudomonas aeruginosa PA01, Staphylococcus aureus MRSA252 and Thermotoga maritima MSB8 (data not shown). A complementary analysis was performed to detect the distribution of the amino acid patterns of RNA MTases across almost 12,000 bacterial genomes in order to disclose possible founder lineages together with orthology and paralogy. In Figure 2, the distribution of the amino acid patterns is observed for the full set of RNA MTases described in E. coli (Table 1). Using violin plots, the large scale information obtained from the similarity networks is better shown. In global terms, the amino acid sequence patterns of RsmC and RsmD MTases are widely spread in bacterial MTases (see the above distribution; 2.5 Similarity Index). Other MTases that have the same profile are $\mathrm{CmoA}, \mathrm{CmoB}, \mathrm{RsmG}, \mathrm{RsmI}$ and TrmN6. The amino acid patterns of these families of MTases were present in other RNA MTases and Ribosome Protein MTases. In addition to the MTase lineages observed in the similarity network analysis, this approach was useful to distinguish unique lineages constituted by RsmE, RImM, the N-terminal domain of the MnmC, TrmD, and RlmK MTases families, thus revealing a very clear profile that supports only orthology with the highest similarity values (Similarity Index $>7.5$ ). Interestingly, members of the SPOUT class of MTases, such as RlmB, TrmH, TrmJ and TrmL, showed a characteristic profile in terms of their amino acid sequence patterns distribution in bacterial genomes. This distribution agrees with paralogy (Similarity Index abundance between $\sim 5$ and 7 ), where duplication and specialization were still detectable at the sequence level $[11,38]$. Likewise, we detected the marked presence of RsmF paralogs, but not in RsmB. Given the poor phylogenetic distribution of RsmF (Figure 1), we hypothesized that such paralogs can perform the RsmF function; therefore, more exhaustive analyses into the phylogenetic relationship and experimental approaches to test the function of these potential new members of the cluster RsmF/B should be addressed in future studies.

\section{Family-specific amino acid models}

Multiple sequence alignments were built for each RNA MTases family (Table 1) using iterative methods. In addition to the conserved pattern of amino acids for each RNA MTases family based on a probabilistic model for amino acid content per site (HMMER3 based analysis), we further analyzed the averaged model of amino acid content per family. We extended our study to other MTases, which are related to RNA MTases according to the similarity networks (Figures 3A-3D), to know whether RNA MTases differ from others acting on substrates other than RNA. After comparing the distribution of amino acids per family through hierarchical clustering, we observed that the entire set of RNA MTases clustered separately from those enzymes acting on non-RNA substrates (Figure 3E). We particularly aimed to disclose the specific amino acids distribution associated with the MTase function. Therefore, we split all the MTases studied into four different groups as follows: 16S MTases, 23S MTases, tRNA MTases, and non-RNA MTases; through multiple pairwise comparisons, we detected the differential amino acid proportions among MTases for amino acids E, I, K, $\mathrm{L}, \mathrm{M}, \mathrm{N}, \mathrm{Q}, \mathrm{R}, \mathrm{S}$, and $\mathrm{V}(\mathrm{p}<0.016)$. As expected, positively charged amino acids $\mathrm{K}$ and $\mathrm{R}$ were found in a higher proportion in all the RNA MTases groups in response to the substrate they modify $(\mathrm{p}<0.00002)$. Charged polar amino acids $\mathrm{N}, \mathrm{S}$ and $\mathrm{Q}$ also showed a high distribution in all the RNA MTases groups $(\mathrm{p}<0.016)$. The $\mathrm{I}, \mathrm{M}, \mathrm{L}$, and $\mathrm{V}$ amino acids had a greater and significantly different distribution in all the non-RNA MTases $(\mathrm{p}<0.001)$. This last observation correlated well with the substrates for these enzymes, such as proteins and coenzyme biosynthesis (biotin and ubiquinone), where hydrophobic interactions can help stabilize enzyme-substrate binding. The negatively charged amino acid such as E, but not D, was differentially found to have high proportions in all the RNA MTases groups $\left(\mathrm{p}<2.0 \times 10^{-9}\right)$. These data were unexpected since a high density of negatively charged amino acids can repel or affect binding with a substrate to present a net electronegative charge. We hypothesized that the relevance of the presence of $E$ in RNA MTases can be explained by counterbalancing the high proportion of positively charged amino acids in structural terms. However, we have no strong evidence to support this notion. Given the good fitted clustering of RNA MTases according to amino acid distribution, we propose that this parameter is a useful criterion to help predict bacterial RNA MTases in addition to structural and sequence evidence.

\section{How MTases evolve}

We tested all the alignments built from the RNA and non-RNA MTase families for approximately 120 empirical amino acid substitution models by using maximum likelihood approaches (see Phylogenetic analyses at Methods). After recovering the model that best explained the amino acid replacement events in each MTase family, we found that all the MTases evolved according to the LG method [82]. This indicates that MTases evolve at different rates along their sequences. This observation is consistent with the fact that most MTase families present a simple architecture consisting of a sole MTase domain. Thus, one or more functions such as substrate recognizing, specificity, 
cofactor binding, and catalysis (functionally and structurally compiled in a unique domain), could evolve differently than others at the MTase inside. Additonally, the model that explains evolution in MTases implies the categorization of sites according to variation level, from invariable to hypervariable sites. Categorization of site variability supports the results stated in the last section and reinforces the idea that the amino acid frequency bias is pivotal during MTases evolution and probably explains their specialization. The evolutionary pattern observed for MTases can be seen in cumulative substitution rate plot in Figure 4C. Using the pangenome information across the more than 360 genomes from the Salmonella enterica strains, we analyzed substitution rates for synonymous and non-synonymous amino acid replacements in one of the MTases presenting an omega value of $(\omega)>1$, suggesting positive selection. In the plot of accumulated substitution rates along the PrmA coding sequence, certain regions or sites where non-synonymous substitutions preferably cluster are clearly observed. This information is particularly relevant and partially explains the vast variability in MTases found as a whole.

\section{Selection to maintain the structure}

We performed multiple sequence alignments among all the MTases analyzed in this study using the amino acid profiles obtained through probabilistic inference and specific algorithms to detect distant homologs (PSI-Coffee based analysis). Figure 5A shows three different similarity regions in the multiple sequence alignment of several related MTases. These similarity regions are not recognized in other Class I MTases that probably conform independent lineages. Data presented in Figure 5A fit the information derived from the study of amino acid substitution model, and these regions correspond to those sites where synonymous substitutions preferentially occur. The relevance of these similarity regions was further considered from the structural point of view (Figure 5B). The similarity regions were identified and highlighted in three different types of MTases analyzed. Although the role of similarity region I is evident and has been previously seen to be involved in AdoMet binding, the role of the other two regions remains unclear. Previous analyses have linked a small motif of region II (N/D-P-P-X) with target nucleotide binding [13], but this motif is present even in some non-RNA MTases such as PrmB and PrmC. When we localized the other two similarity regions into the three-dimensional structures, we realized that they immediately lay adjacent to the first $\beta$-strand comprising the canonical AdoMet binding region (highlighted in blue). Similarity regions II and III predominantly formed the third and fourth $\beta$-strands of the characteristic $\beta$-sheet of the Rossmann Fold. Given their localization in the protein structure, they may play a critical role in structure conformation and stability where the interactions among almost all the amino acids of the similarity regions seem to be evolutionarily conserved. Additionally, we analyzed the amino acid proportions in these three similarity regions for all protein families where they were detected. We found that multiple differences in amino acid content previously detected between RNA and nonRNA MTases were abolished except for Lysine ( $<$ 0.0156). As a consequence, this data also support the idea that Similarity Regions (I-III) evolve in the same manner in all Class I MTases probably as a consequence of their structurally role; therefore, substrate recognizing and binding roles are confined to other regions where amino acid content evolves according to target substrate.

\section{Short-term evolution of RNA methyltransferases (patho-pangenome genetic variability)}

We studied the genetic variation of MTases in eight different human pathogens: Acinetobacter baumannii, Staphylococcus aureus, Pseudomonas aeruginosa, Mycobacterium tuberculosis, Enterococcus faecalis, Enterococcus faecium, Helicobacter pylori, and Salmonella enterica. After an initial examination to detect the MTases encoded in the respective genomes, coding sequences were extracted, aligned and compared in a pair-wise manner. The averaged $\mathrm{dN}$ (non-synonymous rate), $\mathrm{dS}$ (synonymous rate) and $\omega(\mathrm{dN} /$ dS ratio) from the pair-wise comparisons were calculated and used to compare different MTases groups. The $\mathrm{dN}$ and dS from all the MTases gene families found in the eight patho-pangenomes are plotted in Figure 4A. The distribution of the $\mathrm{dS}$ and $\mathrm{dN}$ values was evaluated by calculating linear regression. A correlation among the members belonging to the different MTases groups was observed, and was higher in the 16S rRNA MTases genes. The tRNA and 23S rRNA MTases genes showed similar correlations, with tendency to neutrality in both cases (parallel to the dashed red line). While $\mathrm{dN}$ and $\mathrm{dS}$ values from $16 \mathrm{~S}$ rRNA MTases seem to show a tendency to purifying or stabilizing selection, the non-RNA MTases showed a tendency to positive selection, although they presented a poor correlation coefficient, probably because of the multiple functions included in this group. We found the highest $\omega$ values in the prmA and prmB genes, whose proteins also showed higher connectivity values in the similarity networks (Figures 3A-3D). The distribution of the $\omega$ values is presented in Figure 4B for most of the recurrent MTase gene families found in the pathopangenome analysis. This boxplot shows that prmA, $\operatorname{prm} B$ and $\operatorname{prm} C$, and also $\operatorname{rm} C$, tended to have higher values. Conversely, the genes encoding the RNA MTases showed a distribution with lower $\omega$ values for instance those observed in $r s m B, r s m H$, and rsmI. A detailed view of an MTase evolving under clear positive 


\section{A}

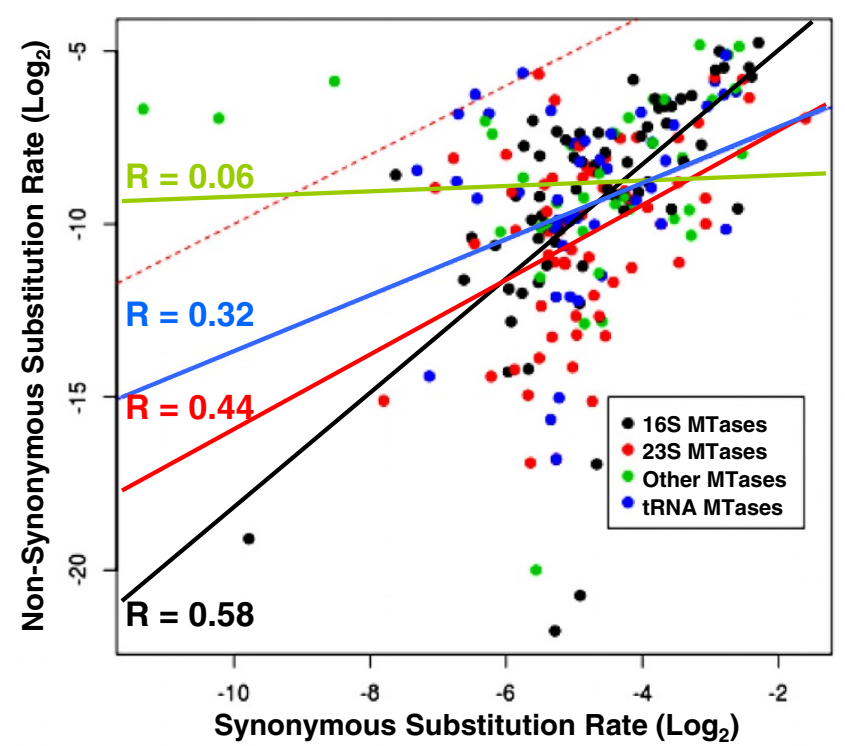

B

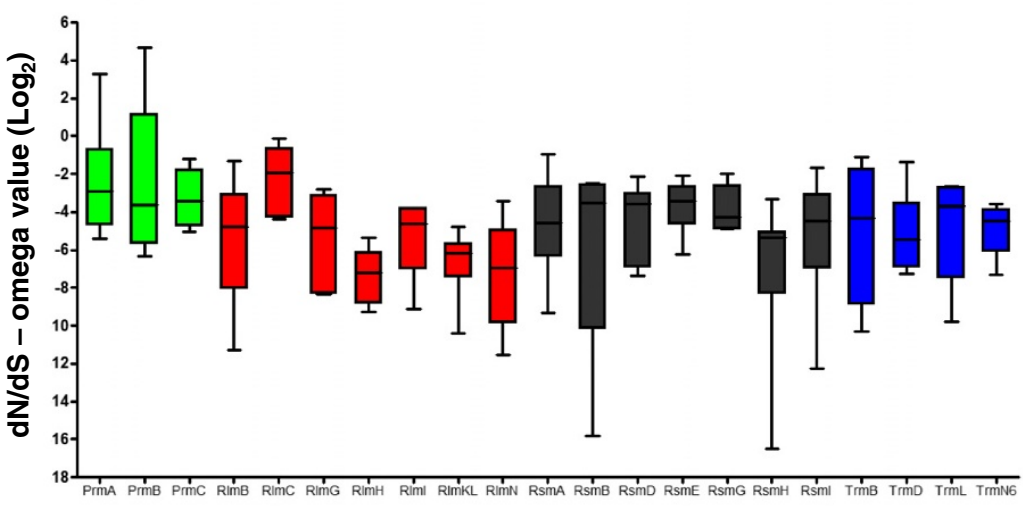

C

PrmA S. enterica

D RImH E. faecium

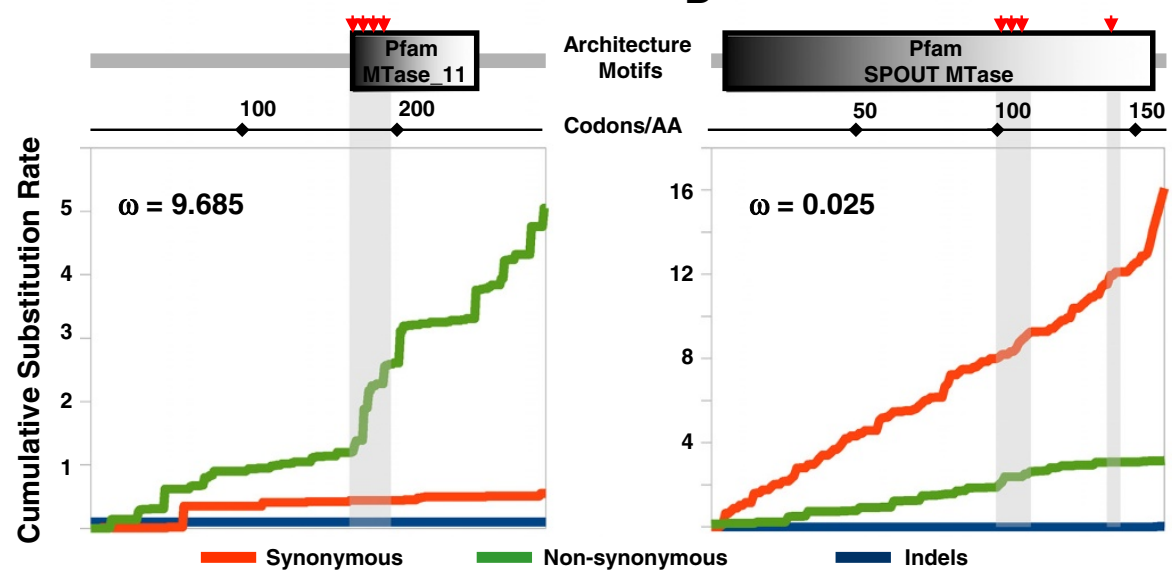

Figure 4 Short-term molecular evolution of the RNA and non-RNA MTases. A - Scatter plot showing the dN and dS Log 2 values for each MTase studied in eight different patho-pangenomes. The MTases were classified according to subtract. The correlation coefficients for each type of MTases were calculated and plotted together with tendency lines. The red dashed line shows the neutrality boundary where the upper values are considered to be under positive selection and the lower one is considered to be under purifying (or stabilizing) selection. B - Boxplot showing the distribution of the $(\omega)$ omega values $\left(\log _{2}\right)$. Categorization of the MTases in agreement with the plot in panel $\mathbf{A}$. Deep view of the synonymous and non-synonymous substitutions on prmA from S. enterica (C), showing one of the highest omega values, and rlmH from E. faecium (D), showing a pattern purifying selection. These plots indicate the exact sites on proteins where the synonymous and non-synonymous substitutions predominantly lie. The critical sites for protein function are highlighted in gray. 


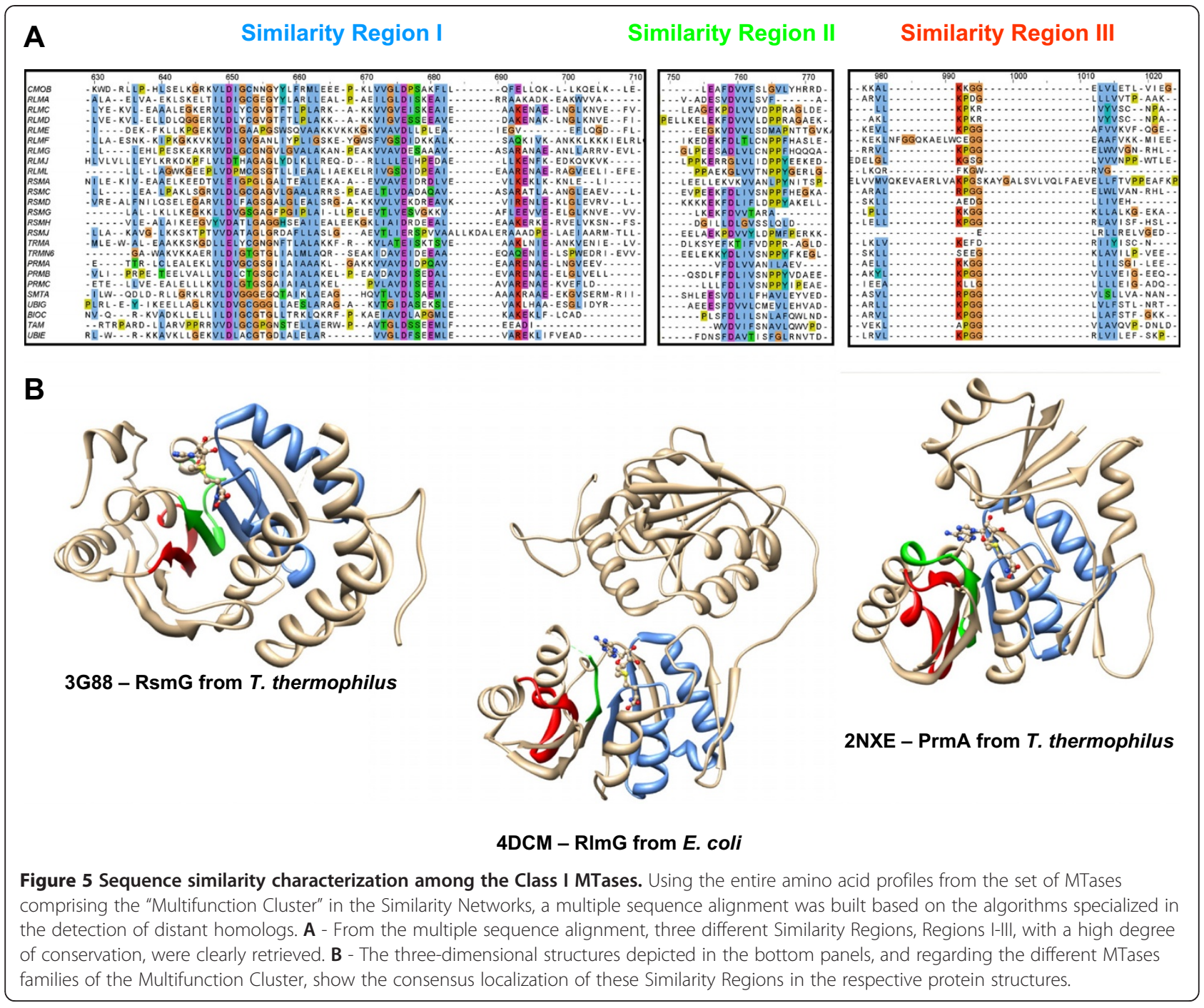

selection and another one evolving under purifying selection is provided in Figures 4C and 4D, respectively. Non-synonymous substitutions found in the prmA gene from $S$. enterica are confined to the amino acid region belonging to the AdoMet binding motif or Similarity Region I (see Figure 5A). In contrast, the MTases genes under the purifying selection (Figure 4D) presented a similar synonymous substitution rate along the gene with no particular concentration of non-synonymous substitutions in any region of the protein.

\section{Genetic variability in antibiotic resistance-associated RNA MTases}

The rRNA MTases, especially those acting on 23S rRNA, showed the lowest $\mathrm{dN}$ values, indicating strong purifying selection (Figure 4A) in human pathogens. As a consequence, we wanted to further explore the cumulative $\mathrm{dN}$ rate in some of these genes with the aim to retrieve evolutionary information from patho-pangenome structure and its possible predisposition to acquire antibiotic resistance. The 16S rRNA MTase RsmG and 23S rRNA MTase RlmN are associated with antibiotic resistance in a wide variety of bacteria, and some of them recurrent human pathogens $[25-27,77,78,83]$. After analyzing the pattern of the cumulative $\mathrm{dS}$ and $\mathrm{dN}$ rates along the $r s m G$ and $r \operatorname{lm} N$ genes, we found that $\omega$ values were higher in $r \operatorname{sm} G$ than in $r \operatorname{lm} N$ in all the pangenomes analyzed (Additional file 1: Figure S1). This difference was most obvious in E. faecalis, where $r \operatorname{lm} N$ had almost null non-synonymous substitutions (>300-fold). The cumulative dS rate pattern was similar in both, indicating that synonymous substitutions occur at the same rate along the respective genomes. When codon hotspot sites for protein inactivation are taken into account [27,84-87], as well as Similarity Regions among Class I MTases described here (Figure 5A), difference among the cumulative $\mathrm{dN}$ rates between $r \operatorname{lm} N$ and $r s m G$ indicates that this last could undergo a selection which would affect pivotal sites for protein function, 
essentially where AdoMet binding underlies. A site-by-site analysis of cumulative synonymous and non-synonymous substitutions in the rsmG AdoMet binding site showed that non-synonymous substitutions fall outside critical sites for protein function (Additional file 2: Figure S2).

\section{Conclusions}

The study of RNA MTases can help to understand their role in translation. Given the enormous variability among the RNA MTases, their evolutionary relationship is unclear. Here we presented data to support the notion that several MTases emerge from one common ancestor. Nevertheless, we could not identify the ancestral sequence. We reviewed the entire set of RNA MTases described for Escherichia coli, and we disclosed a core set of RNA MTases in Eubacteria by studying phylogenetic profiles in different phyla. We identified approximately 13 RNA MTase families that are highly conserved across bacterial species which probably represent the core of methylations for the proper function of tRNA and rRNA. From the amino acid and DNA sequences analyses, we showed that most Class I RNA MTases are related to Ribosomal Protein MTases, such as PrmA, PrmB, and PrmC, as well as other MTases that act in cofactor/vitamin biosynthesis. The Prm proteins show many links with RNA MTases (Figure 3) and their high proportion of non-synonymous substitutions could support their role as a founder lineage of Class I MTases included in the "Multifunction Cluster" defined here. We could identify unique lineages through massive sequence comparisons using the genomic information of almost 12,000 bacterial genomes. The RNA MTases that seem to be unique in sequence terms are RsmE, RlmK, TrmD, RlmM, RlmN and the N-terminal domain of the bi-functional MnmC MTase. These families, together with the three different clusters evidenced by our similarity network analysis, indicate that RNA MTases diversity can be explained, at least from the emergence of nine MTase lineages. Although we have not taken into account other important groups of enzymes, such as DNA MTases, our data indicates that multiple emergence events explain the vast diversity of MTases. We also found that despite the sequence relationships, RNA MTases, and those acting in different molecules; diverge in the amino acid content, a fact that well matches the function associated with different MTases. Members of the "Multifunction Cluster" present three clear similarity regions (Figure 5). By combining the intensive amino acid sequence, the evolutionary model prediction and the molecular evolution analyses provided evidence supporting the idea that AdoMet-dependent Class I MTases are under strong purifying selection to retain the protein structure and cofactor binding site. We present a pathopangenome molecular evolution analysis to define the short-term evolution pattern of a large set of RNA MTases and non-RNA MTases for the purpose of linking their evolution with pathogenesis. The acquisition and development of antibiotic resistance is a common feature among persistent infections. This has been strongly linked to some methylations in rRNA $[27,77,78]$, and the mechanisms for progression from low level resistance to a high level is still unclear $[27,28]$. We found that rRNA MTAses evolve close to neutrality with very low non-synonymous substitution rates. We found that human pathogens are prone to accumulate non-synonymous substitutions outside critical sites of RNA MTases (Additional file 2: Figure S2). Based on these data, RNA MTases in human pathogens seem to follow patterns of evolution observed for MTases. This pattern is widespread among MTases and even in those associated to mutation-dependent mechanism to acquire and develop antibiotic resistance. Data obtained from different approaches used in this study fit well patterns of variation observed for bacterial AdoMetdependent non-coding RNA MTases, and they may represent a response to substrate specialization but retaining ancient functional modules.

\section{Methods}

\section{Sequence analyses}

The phylogenetic distribution and relationships of RNA MTases were studied by downloading a set of more than 3,000 protein sequences grouped into 34 families based on the full core of RNA MTases functionally characterized to act in E. coli rRNAs and tRNAs [7,9,18,27,34,39-69] (Table 1). Using the amino acid sequences of $E$. coli RNA MTases as queries [88], a Blastp search against the non redundant Reference Sequences Database at NCBI [89] was conducted with default parameters (http://blast.ncbi.nlm. nih.gov/Blast.cgi) [90]. We explored the phylogenetic distribution of the RNA MTases homologs in major bacteria groups (i.e., Acidobacteria, Actinobacteria, Bacteroidetes, Chloroflexi, Chlamydiae, Cyanobacteria, Deinococci, Firmicutes, Fusobacteria, Proteobacteria, Spirochaetes, Tenericutes, and Thermotogae). Based on pair-wise comparisons with an alignment coverage of $>75 \%$ and an alignment score of $>60$ bits, we retrieved more than 3,000 different sequences representative of the diversity of RNA MTases for each bacterial phylum. Each family of RNA MTases was then aligned using the Probcons software, v1.12, with 1,000 passes of iterative refinement [91], followed by filtering for gaps.

\section{Amino acid profiles}

High quality alignments were used to built respective amino acid profiles were constructed using the HMMER3 algorithm and default parameters [92]. The protein architecture was examined using the respective HMM-based amino acid profiles and the SMART server [93]. The averaged amino acid distribution per family was analyzed 
using hierarchical clustering. Consequently, the heatmaps of amino acid composition were generated using the gplots library in $\mathrm{R}$ [94] with previous $\log 2$-transformation of frequencies and clustering with a complete method and euclidean distance. The RNA MTase networks, based on probabilistic inference methods and sequence relationships among RNA MTases, were constructed for model organisms, such as Escherichia coli K12 (GenBank id, NC_000913) and Bacillus subtilis 168 (NC_00949), using Biolayout Express 3D and the Markov Clustering Algorithm (MCL) [95]. The clustering of nodes was performed for Mycobacterium tuberculosis H37Rv (NC_018143), Pseudomonas aeruginosa PA01 (NC_002516), Staphylococcus aureus MRSA252 (NC_002952), Thermotoga maritima MSB8 (NC_021214). As a result, the amino acids profiles based on Hidden Markov Models (HMM) for the 34 RNA MTases families and the nine additional families of E. coli non-RNA MTases (BioB, BioC, PrmA, PrmB, PrmC, SmtA, Tam, UbiE, and UbiG) were compiled and indexed in an HMM database using the hmmpress algorithm contained in the HMMER3 package. A search for the proteins related to the MTases proteins was done using the hmmscan algorithm (HMMER3 package) with a threshold score of $>25$. Proteins sharing a sequence similarity against the MTase profiles compiled in the HMM database were ranked according to a normalized Similarity Index $=\log _{2}[(\mathrm{Lt} / \mathrm{Lp}) \times \mathrm{S}]$, where $\mathrm{Lt}$ is equal to the length of the sequence aligned in the target, $\mathrm{Lp}$ is the total length of the query amino acid profile, and $\mathrm{S}$ is the alignment score. This Similarity Index was used as a measurement of the sequence relationships among the MTases reflecting the edges in the protein networks.

\section{Phylogenetic analyses}

Relationships among the RNA MTAses were analyzed by two approaches. First, occurrence probabilities for all the amino acids in each MTase family. The HMM profiles built with the HMMER3 software. All probabilities were set as variables in a similarity matrix, and a dendrogram was constructed using the UPGMA algorithm with Pearson's coefficient and 100 bootstrap replicates on the following web server: http://genomes.urv.cat/ UPGMA/index.php [96]. The multiple pair-wise comparisons made among the RNA MTase groups were calculated in R v3.0 (http://www.r-project.org/) and an ANOVA test with Bonferroni correction was used. The second approach performed to disclose the evolutionary model for each RNA MTase family analyzed. Likelihoods for 120 empirical models (containing 15 different matrices) implemented in ProtTest v3.3 were calculated [97-99]. The best model was selected according to the smallest corrected Akaike Information Criterion (AICc). The Similarity Regions among MTases was obtained by the multiple sequence alignment of the respective amino acid profiles obtained from HMMER3 and using iterative algorithms for distantly related sequences (PSI -Coffee at T-Coffee web server, http://www.tcoffee.org) [100,101].

\section{Genome-scale analysis of bacterial pathogens}

Presence of different RNA MTases and related proteins was massively detected in almost 12,000 fully-sequenced bacterial genomes publicly available in the Pathosystems Resource Integration Center (PATRIC). Approximately 50 million encoded proteins were tested to match the RNA MTases using probabilistic inference methods, as previously stated. The alignment hits and respective Similarity Index were clustered according to RNA MTase similarity. Then the violin density plots were drawn in $\mathrm{R} \mathrm{v3.0}$ (http://www.r-project.org/) and the ggplot2 package [102]. According to the Similarity Index distribution among the different protein families, the hits showing a Similarity Index higher than 7.5 were selected as true orthologs, whereas those hits showing a Similarity Index lower than 5 and higher than 2.5 were considered to be proteins that were phylogenetically related to the RNA MTases, based on the criteria of at least 35 aa in length and a score $~ 30$. The alignment hits showing a Similarity Index higher than 5 were and lower than 7 were selected as the potential paralogs. These latter proteins, which exhibited a potential paralogy with certain RNA MTases, were extracted and functional prediction was assessed according to the sequence, motifs, and architecture criteria.

\section{Genetic variability in patho-pangenomes}

The intra-species molecular evolution of the RNA MTases in human pathogens was investigated by analyzing the genetic variability in these genes in almost 2,000 genomes. Consequently, the coding sequences for all the RNA MTases studied, when presented, were extracted from pangenomes from eight common human pathogens: Acinetobacter baumannii (186 genomes), Staphylococcus aureus (438 genomes), Pseudomonas aeruginosa (47 genomes), Mycobacterium tuberculosis (75 genomes), Enterococcus faecalis (271 genomes), Enterococcus faecium (229 genomes), Helicobacter pylori (243 genomes) and Salmonella enterica (393 genomes). They were respectively aligned using iterative and accurate methods $[103,104]$. The synonymous and non-synonymous substitution rates were calculated in a pair-wise fashion using SNAP calculator v1.1 [105] and by correcting transitional substitutions [106]. As a result, the synonymous and non-synonymous substitution rates and the proportions for the transitional substitutions were obtained and used for the comparisons made among the MTases families. Linear regression and multiple pair-wise comparisons were done among the RNA MTase groups, and were calculated in R v3.0 (http://www.r-project.org/) using an ANOVA test with Bonferroni correction. 


\section{Additional files}

Additional file 1: Figure S1. The Cumulative Substitution Rate plots for genes $r / m N$ and $r \mathrm{mG}$. A comparative analysis done with the genes and pathogens for the distribution of the synonymous and non-synonymous substitutions in genes $r / m N$ and $r s m G$ is shown. Those genes are well known to be associated to antibiotic resistance. Critical sites for the protein function are highlighted in gray. The correlation between the high accumulation of the non-synonymous substitutions and hotspots for the functional inactivation of RsmG are more clearly inferred.

Additional file 2: Figure S2. Close view for Cumulative Substitution Rate in rsmG. Two plots showing the distribution of the synonymous and non-synonymous substitutions at amino acid sequence level in rsm G genes from $H$. pylori and $P$. aeruginosa. Red lines show cumulative synonymous substitutions and green lines show non-synonymous substitutions. Hotspots for protein inactivation (gray shaded amino acid positions) were compiled from $[27,85]$.

\section{Competing interests}

The authors declare that they have no competing interests.

\section{Author's contribution}

ABP designed this study. JMR and SCB carried out the sequence and phylogenetic analyses. MC and JDP assisted sequence and phylogenetic analyses and computing performance. ABP and JMR worked in manuscript preparation. All authors read and approved the final manuscript.

\section{Acknowledgements}

This work was supported by the Colombian Agency for Science, Technology, and Innovation - COLCIENCIAS; and the National Fund for Science, Technology, and Innovation "Francisco José de Caldas" [grant 5817-56934856 to ABP]. The authors would like to thank the Editors and peer reviewers for their constructive suggestions to improve this manuscript.

\section{Author details}

1Bioinformatics Analysis Group - GABi, Centro de Investigación y Desarrollo en Biotecnología - CIDBIO, 111221 Bogotá, D.C, Colombia. ${ }^{2}$ GEBIOMIC Group, Faculty of Life Sciences, Universidad de Antioquia, Medellín, Colombia. ${ }^{3}$ APOLO Computing Center. EAFIT, Medellín, Colombia.

Received: 10 April 2014 Accepted: 2 July 2014

Published: 10 July 2014

\section{References}

1. Agris PF: Bringing order to translation: the contributions of transfer RNA anticodon-domain modifications. EMBO Rep 2008, 9(7):629-635.

2. Helm M: Post-transcriptional nucleotide modification and alternative folding of RNA. Nucleic Acids Res 2006, 34(2):721-733.

3. Grosjean H: Fine tuning of RNA functions by modification and editing. In Topics in Current Genetics. 12th edition. Edited by Hohmann S. New York: Springer Verlag; 2005.

4. Decatur WA, Fournier MJ: rRNA modifications and ribosome function. Trends Biochem Sci 2002, 27(7):344-351.

5. Björk GR, Hagervall TG: Transfer RNA modification. In EcoSal-Escherichia coli and Salmonella: cellular and molecular biology. Edited by Böck RCl, Kaper JB, Neidhardt FC, Nyström T, Rudd KE, Squires CL. Washington, D.C: ASM Press; 2005.

6. Ofengand J, Campo M: Modified Nucleosides of Escherichia coli Ribosomal RNA. In EcoSal-Escherichia coli and Salmonella: cellular and molecular biology. Edited by Böck RCl, Kaper JB, Neidhardt FC, Nyström T, Rudd KE, Squires CL. Washington, D.C: ASM Press; 2005

7. Benitez-Paez A, Villarroya M, Armengod ME: The Escherichia coli RImN methyltransferase is a dual-specificity enzyme that modifies both rRNA and tRNA and controls translational accuracy. RNA 2012, 18(10):1783-1795.

8. Demirci H, Larsen LH, Hansen T, Rasmussen A, Cadambi A, Gregory ST, Kirpekar F, Jogl G: Multi-site-specific 16S rRNA methyltransferase RsmF from Thermus thermophilus. RNA 2010, 16(8):1584-1596.

9. Ranaei-Siadat E, Fabret C, Seijo B, Dardel F, Grosjean H, Nonin-Lecomte S: RNA-methyltransferase TrmA is a dual-specific enzyme responsible for
C5-methylation of uridine in both tmRNA and tRNA. RNA Biol 2013, 10(4):572-578

10. Desmolaize B, Fabret C, Bregeon D, Rose S, Grosjean H, Douthwaite S: A single methyltransferase YefA (RImCD) catalyses both m5U747 and m5U1939 modifications in Bacillus subtilis 23S rRNA. Nucleic Acids Res 2011, 39(21):9368-9375.

11. Anantharaman V, Koonin EV, Aravind L: SPOUT: a class of methyltransferases that includes spoU and trmD RNA methylase superfamilies, and novel superfamilies of predicted prokaryotic RNA methylases. J Mol Microbiol Biotechnol 2002, 4(1):71-75.

12. Anantharaman V, Koonin EV, Aravind L: Comparative genomics and evolution of proteins involved in RNA metabolism. Nucleic Acids Res 2002, 30(7):1427-1464.

13. Schubert $H L$, Blumenthal RM, Cheng $X$ : Many paths to methyltransfer: a chronicle of convergence. Trends Biochem Sci 2003, 28(6):329-335.

14. Martin JL, MCMillan FM: SAM (dependent) I AM: the S-adenosylmethioninedependent methyltransferase fold. Curr Opin Struct Biol 2002, 12(6):783-793

15. O'Farrell HC, Xu Z, Culver GM, Rife JP: Sequence and structural evolution of the KsgA/Dim1 methyltransferase family. BMC Res Notes 2008, 1:108.

16. Xu Z, O'Farrell HC, Rife JP, Culver GM: A conserved rRNA methyltransferase regulates ribosome biogenesis. Nat Struct Mol Biol 2008, 15(5):534-536.

17. Hagervall TG, Tuohy TM, Atkins JF, Bjork GR: Deficiency of 1-methylguanosine in tRNA from Salmonella typhimurium induces frameshifting by quadruplet translocation. J Mol Biol 1993, 232(3):756-765.

18. Kimura S, Suzuki T: Fine-tuning of the ribosomal decoding center by conserved methyl-modifications in the Escherichia coli 16S rRNA. Nucleic Acids Res 2010, 38(4):1341-1352.

19. Li JN, Bjork GR: 1-Methylguanosine deficiency of tRNA influences cognate codon interaction and metabolism in Salmonella typhimurium. J Bacteriol 1995, 177(22):6593-6600

20. Urbonavicius J, Qian Q, Durand JM, Hagervall TG, Bjork GR: Improvement of reading frame maintenance is a common function for several tRNA modifications. EMBO J 2001, 20(17):4863-4873.

21. O'Dwyer K, Watts JM, Biswas S, Ambrad J, Barber M, Brule H, Petit C, Holmes DJ, Zalacain M, Holmes WM: Characterization of Streptococcus pneumoniae TrmD, a tRNA methyltransferase essential for growth. J Bacterio/ 2004, 186(8):2346-2354.

22. Helser TL, Davies JE, Dahlberg JE: Mechanism of kasugamycin resistance in Escherichia coli. Nat New Biol 1972, 235(53):6-9.

23. Sparling PF, Ikeya $Y$, Elliot D: Two genetic loci for resistance to kasugamycin in Escherichia coli. J Bacterio/ 1973, 113(2):704-710.

24. Zimmermann RA, Ikeya Y, Sparling PF: Alteration of ribosomal protein S4 by mutation linked to kasugamycin-resistance in Escherichia coli. Proc Natl Acad Sci U S A 1973, 70(1):71-75

25. Nishimura K, Hosaka T, Tokuyama S, Okamoto S, Ochi K: Mutations in rsmG, encoding a $16 \mathrm{~S}$ rRNA methyltransferase, result in low-level streptomycin resistance and antibiotic overproduction in Streptomyces coelicolor A3 (2). J Bacteriol 2007, 189(10):3876-3883.

26. Nishimura K, Johansen SK, Inaoka T, Hosaka T, Tokuyama S, Tahara Y, Okamoto S, Kawamura F, Douthwaite S, Ochi K: Identification of the RsmG methyltransferase target as $16 \mathrm{~S}$ rRNA nucleotide G527 and characterization of Bacillus subtilis rsmG mutants. J Bacterio/ 2007, 189 (16):6068-6073.

27. Okamoto S, Tamaru A, Nakajima C, Nishimura K, Tanaka Y, Tokuyama S, Suzuki Y, Ochi K: Loss of a conserved 7-methylguanosine modification in 16S rRNA confers low-level streptomycin resistance in bacteria. Mol Microbiol 2007, 63(4):1096-1106.

28. Ochi K, Kim JY, Tanaka Y, Wang G, Masuda K, Nanamiya H, Okamoto S, Tokuyama S, Adachi Y, Kawamura F: Inactivation of KsgA, a 16S rRNA methyltransferase, causes vigorous emergence of mutants with high-level kasugamycin resistance. Antimicrob Agents Chemother 2009, 53(1):193-201.

29. Galimand M, Courvalin P, Lambert T: Plasmid-mediated high-level resistance to aminoglycosides in Enterobacteriaceae due to 16S rRNA methylation. Antimicrob Agents Chemother 2003, 47(8):2565-2571.

30. Gonzalez-Zorn B, Catalan A, Escudero JA, Dominguez L, Teshager T, Porrero C, Moreno MA: Genetic basis for dissemination of armA. J Antimicrob Chemother 2005, 56(3):583-585.

31. Gonzalez-Zorn B, Teshager T, Casas M, Porrero MC, Moreno MA, Courvalin P, Dominguez L: armA and aminoglycoside resistance in Escherichia coli. Emerg Infect Dis 2005, 11(6):954-956. 
32. Schwarz S, Werckenthin C, Kehrenberg C: Identification of a plasmid-borne chloramphenicol-florfenicol resistance gene in Staphylococcus sciuri. Antimicrob Agents Chemother 2000, 44(9):2530-2533.

33. Weisblum B: Erythromycin resistance by ribosome modification Antimicrob Agents Chemother 1995, 39(3):577-585.

34. Golovina AY, Dzama MM, Osterman IA, Sergiev PV, Serebryakova MV, Bogdanov AA, Dontsova OA: The last rRNA methyltransferase of E. coli revealed: the yhiR gene encodes adenine-N6 methyltransferase specific for modification of A2030 of 23S ribosomal RNA. RNA 2012 18(9):1725-1734.

35. Zhang H, Wan H, Gao ZQ, Wei Y, Wang WJ, Liu GF, Shtykova EV, Xu JH, Dong $\mathrm{YH}$ : Insights into the catalytic mechanism of $16 \mathrm{~S}$ rRNA methyltransferase RsmE (m(3)U1498) from crystal and solution structures. J Mol Biol 2012, 423(4):576-589.

36. Boal AK, Grove TL, McLaughlin MI, Yennawar NH, Booker SJ, Rosenzweig AC: Structural basis for methyl transfer by a radical SAM enzyme. Science 2011, 332(6033):1089-1092.

37. Sunita S, Tkaczuk KL, Purta E, Kasprzak JM, Douthwaite S, Bujnicki JM, Sivaraman J: Crystal structure of the Escherichia coli 23S rRNA: m5C methyltransferase RIml (YccW) reveals evolutionary links between RNA modification enzymes. J Mol Biol 2008, 383(3):652-666.

38. Tkaczuk KL, Dunin-Horkawicz S, Purta E, Bujnicki JM: Structural and evolutionary bioinformatics of the SPOUT superfamily of methyltransferases. BMC Bioinformatics 2007, 8:73

39. Gustafsson C, Persson BC: Identification of the rrmA gene encoding the $23 \mathrm{~S}$ rRNA m1G745 methyltransferase in Escherichia coli and characterization of an m1G745-deficient mutant. J Bacteriol 1998, 180(2):359-365.

40. Lovgren JM, Wikstrom PM: The rlmB gene is essential for formation of Gm2251 in 23S rRNA but not for ribosome maturation in Escherichia coli. J Bacterio/ 2001, 183(23):6957-6960

41. Madsen CT, Mengel-Jorgensen J, Kirpekar F, Douthwaite S: Identifying the methyltransferases for m(5)U747 and m(5)U1939 in 23S rRNA using MALDI mass spectrometry. Nucleic Acids Res 2003, 31(16):4738-4746.

42. Agarwalla S, Kealey JT, Santi DV, Stroud RM: Characterization of the $23 \mathrm{~S}$ ribosomal RNA m5U1939 methyltransferase from Escherichia coli. J Biol Chem 2002, 277(11):8835-8840

43. Caldas $T$, Binet $E$, Bouloc $P$, Richarme $G$ : Translational defects of Escherichia coli mutants deficient in the Um(2552) 23S ribosomal RNA methyltransferase RrmJ/FTSJ. Biochem Biophys Res Commun 2000, 271(3):714-718.

44. Purta E, Kaminska KH, Kasprzak JM, Bujnicki JM, Douthwaite S: YbeA is the m3Psi methyltransferase RImH that targets nucleotide 1915 in 23S rRNA. RNA 2008, 14(10):2234-2244.

45. Sergiev PV, Lesnyak DV, Bogdanov AA, Dontsova OA: Identification of Escherichia coli $\mathrm{m} 2 \mathrm{G}$ methyltransferases: Il. the ygjO gene encodes a methyltransferase specific for G1835 of the 23 S rRNA. J Mol Biol 2006, 364(1):26-31.

46. Purta $\mathrm{E}, \mathrm{O}^{\prime}$ Connor $\mathrm{M}$, Bujnicki JM, Douthwaite $\mathrm{S}$ : $\mathrm{YccW}$ is the $\mathrm{m} 5 \mathrm{C}$ methyltransferase specific for $23 \mathrm{~S}$ rRNA nucleotide 1962. J Mol Biol 2008 383(3):641-651.

47. Lesnyak DV, Sergiev PV, Bogdanov AA, Dontsova OA: Identification of Escherichia coli m2G methyltransferases: I. the ycbY gene encodes a methyltransferase specific for G2445 of the 23 S rRNA. J Mol Biol 2006 364(1):20-25.

48. Kimura S, Ikeuchi Y, Kitahara K, Sakaguchi Y, Suzuki T, Suzuki T: Base methylations in the double-stranded RNA by a fused methyltransferase bearing unwinding activity. Nucleic Acids Res 2012, 40(9):4071-4085.

49. Wang KT, Desmolaize B, Nan J, Zhang XW, Li LF, Douthwaite S, Su XD Structure of the bifunctional methyltransferase YcbY (RImKL) that adds the m7G2069 and m2G2445 modifications in Escherichia coli 23S rRNA. Nucleic Acids Res 2012, 40(11):5138-5148.

50. Purta E, O'Connor M, Bujnicki JM, Douthwaite S: YgdE is the 2'-O-ribose methyltransferase RImM specific for nucleotide C2498 in bacterial 235 rRNA. Mol Microbiol 2009, 72(5):1147-1158.

51. Toh SM, Xiong L, Bae T, Mankin AS: The methyltransferase $\mathrm{YfgB} / \mathrm{RlmN}$ is responsible for modification of adenosine 2503 in 23S rRNA. RNA 2008, 14(1):98-106.

52. van Buul CP, van Knippenberg PH: Nucleotide sequence of the ksgA gene of Escherichia coli: comparison of methyltransferases effecting dimethylation of adenosine in ribosomal RNA. Gene 1985, 38(1-3):65-72.
53. Gu XR, Gustafsson C, Ku J, Yu M, Santi DV: Identification of the 16S rRNA m5C967 methyltransferase from Escherichia coli. Biochemistry 1999, 38 (13):4053-4057.

54. Tscherne JS, Nurse K, Popienick P, Michel H, Sochacki M, Ofengand J: Purification, cloning, and characterization of the 16S RNA m5C967 methyltransferase from Escherichia coli. Biochemistry 1999, 38(6):1884-1892.

55. Tscherne JS, Nurse K, Popienick P, Ofengand J: Purification, cloning, and characterization of the 16 S RNA m2G1207 methyltransferase from Escherichia coli. J Biol Chem 1999, 274(2):924-929.

56. Lesnyak DV, Osipiuk J, Skarina T, Sergiev PV, Bogdanov AA, Edwards A, Savchenko A, Joachimiak A, Dontsova OA: Methyltransferase that modifies guanine 966 of the $16 \mathrm{~S}$ rRNA: functional identification and tertiary structure. J Biol Chem 2007, 282(8):5880-5887.

57. Basturea GN, Rudd KE, Deutscher MP: Identification and characterization of RsmE, the founding member of a new RNA base methyltransferase family. RNA 2006, 12(3):426-434.

58. Andersen NM, Douthwaite $\mathrm{S}$ : YebU is a $\mathrm{m} 5 \mathrm{C}$ methyltransferase specific for 16 S rRNA nucleotide 1407. J Mol Biol 2006, 359(3):777-786.

59. Basturea GN, Dague DR, Deutscher MP, Rudd KE: YhiQ is RsmJ, the methyltransferase responsible for methylation of G1516 in 16S rRNA of E. coli. J Mol Biol 2012, 415(1):16-21.

60. Ny T, Bjork GR: Cloning and restriction mapping of the trmA gene coding for transfer ribonucleic acid (5-methyluridine)-methyltransferase in Escherichia coli K-12. J Bacterio/ 1980, 142(2):371-379.

61. De Bie LG, Roovers M, Oudjama Y, Wattiez R, Tricot C, Stalon V, Droogmans $L$, Bujnicki JM: The yggH gene of Escherichia coli encodes a tRNA (m7G46) methyltransferase. J Bacteriol 2003, 185(10):3238-3243.

62. Hjalmarsson KJ, Bystrom AS, Bjork GR: Purification and characterization of transfer RNA (guanine-1) methyltransferase from Escherichia coli. J Biol Chem 1983, 258(2):1343-1351.

63. Persson BC, Jager G, Gustafsson C: The spoU gene of Escherichia coli, the fourth gene of the spoT operon, is essential for tRNA (Gm18) 2'-Omethyltransferase activity. Nucleic Acids Res 1997, 25(20):4093-4097.

64. Purta E, van Vliet F, Tkaczuk KL, Dunin-Horkawicz S, Mori H, Droogmans L, Bujnicki JM: The yfhQ gene of Escherichia coli encodes a tRNA:Cm32/ Um32 methyltransferase. BMC Mol Biol 2006, 7:23.

65. Benitez-Paez A, Villarroya M, Douthwaite S, Gabaldon T, Armengod ME: YibK is the 2'-O-methyltransferase $T r m L$ that modifies the wobble nucleotide in Escherichia coli tRNA(Leu) isoacceptors. RNA 2010, 16(11):2131-2143.

66. Golovina AY, Sergiev PV, Golovin AV, Serebryakova MV, Demina I, Govorun VM, Dontsova OA: The yfiC gene of E. coli encodes an adenine-N6 methyltransferase that specifically modifies A37 of tRNA1Val(cmo5UAC). RNA 2009, 15(6):1134-1141

67. Bujnicki JM, Oudjama Y, Roovers M, Owczarek S, Caillet J, Droogmans L: Identification of a bifunctional enzyme $\mathrm{MnmC}$ involved in the biosynthesis of a hypermodified uridine in the wobble position of tRNA. RNA 2004, 10(8):1236-1242

68. Hagervall TG, Bjork GR: Genetic mapping and cloning of the gene (trmC) responsible for the synthesis of tRNA ( $\mathrm{mnm} 5 \mathrm{~s} 2 \mathrm{U})$ methyltransferase in Escherichia coli K12. Mol Gen Genet 1984, 196(2):201-207.

69. Nasvall SJ, Chen P, Bjork GR: The modified wobble nucleoside uridine-5oxyacetic acid in tRNAPro(cmo5UGG) promotes reading of all four proline codons in vivo. RNA 2004, 10(10):1662-1673.

70. Ogle JM, Brodersen DE, Clemons WM Jr, Tarry MJ, Carter AP, Ramakrishnan $\checkmark$ : Recognition of cognate transfer RNA by the 30 S ribosomal subunit. Science 2001, 292(5518):897-902.

71. Qin D, Liu Q, Devaraj A, Fredrick K: Role of helix 44 of 165 rRNA in the fidelity of translation initiation. RNA 2012, 18(3):485-495

72. von Ahsen U, Noller HF: Identification of bases in $16 \mathrm{~S}$ rRNA essential for tRNA binding at the 30 S ribosomal P site. Science 1995, 267(5195):234-237.

73. Liiv A, Karitkina D, Maivali U, Remme J: Analysis of the function of E. coli 23S rRNA helix-loop 69 by mutagenesis. BMC Mol Biol 2005, 6:18.

74. Spahn CM, Remme J, Schafer MA, Nierhaus KH: Mutational analysis of two highly conserved UGG sequences of $23 \mathrm{~S}$ rRNA from Escherichia coli. J Biol Chem 1996, 271(51):32849-32856.

75. Dailidiene D, Bertoli MT, Miciuleviciene J, Mukhopadhyay AK, Dailide G, Pascasio MA, Kupcinskas L, Berg DE: Emergence of tetracycline resistance in Helicobacter pylori: multiple mutational changes in $16 \mathrm{~S}$ ribosomal DNA and other genetic loci. Antimicrob Agents Chemother 2002, 46(12):3940-3946. 
76. Liu M, Kirpekar F, Van Wezel GP, Douthwaite S: The tylosin resistance gene tlrB of Streptomyces fradiae encodes a methyltransferase that targets G748 in 23S rRNA. Mol Microbiol 2000, 37(4):811-820.

77. Gao W, Chua K, Davies JK, Newton HJ, Seemann T, Harrison PF, Holmes NE, Rhee HW, Hong J, Hartland EL, Stinear TP, Howden BP: Two novel point mutations in clinical Staphylococcus aureus reduce linezolid susceptibility and switch on the stringent response to promote persistent infection. PLOS Pathog 2010, 6(6):e1000944.

78. LaMarre JM, Howden BP, Mankin AS: Inactivation of the indigenous methyltransferase RImN in Staphylococcus aureus increases linezolid resistance. Antimicrob Agents Chemother 2011, 55(6):2989-2991.

79. Kehrenberg C, Schwarz S, Jacobsen L, Hansen LH, Vester B: A new mechanism for chloramphenicol, florfenicol and clindamycin resistance: methylation of 23S ribosomal RNA at A2503. Mol Microbiol 2005, 57(4):1064-1073.

80. Long KS, Poehlsgaard J, Kehrenberg C, Schwarz S, Vester B: The Cfr rRNA methyltransferase confers resistance to Phenicols, Lincosamides, Oxazolidinones, Pleuromutilins, and Streptogramin A antibiotics. Antimicrob Agents Chemother 2006, 50(7):2500-2505.

81. Urbonavicius J, Skouloubris S, Myllykallio H, Grosjean H: Identification of novel gene encoding a flavin-dependent tRNA:m5U methyltransferase in bacteria-evolutionary implications. Nucleic Acids Res 2005, 33(13):3955-3964

82. Le $S Q$, Gascuel O: An improved general amino acid replacement matrix. Mol Biol Evol 2008, 25(7):1307-1320.

83. Gregory ST, Demirci H, Belardinelli R, Monshupanee T, Gualerzi C, Dahlberg AE, Jogl G: Structural and functional studies of the Thermus thermophilus $16 \mathrm{~S}$ rRNA methyltransferase RsmG. RNA 2009, 15(9):1693-1704.

84. Atkinson GC, Hansen LH, Tenson T, Rasmussen A, Kirpekar F, Vester B: Distinction between the Cfr methyltransferase conferring antibiotic resistance and the housekeeping RImN methyltransferase. Antimicrob Agents Chemother 2013, 57(8):4019-4026.

85. Benitez-Paez A, Villarroya M, Armengod ME: Regulation of expression and catalytic activity of Escherichia coli RsmG methyltransferase. RNA 2012, 18(4):795-806

86. McCusker KP, Medzihradszky KF, Shiver AL, Nichols RJ, Yan F, Maltby DA, Gross CA, Fujimori DG: Covalent intermediate in the catalytic mechanism of the radical S-adenosyl-L-methionine methyl synthase RImN trapped by mutagenesis. J Am Chem Soc 2012, 134(43):18074-18081.

87. Benitez-Paez A, Cardenas-Brito S, Corredor M, Villarroya M, Armengod ME: Impairing methylations at ribosome RNA, a point mutation-dependent strategy for aminoglycoside resistance: the rsmG case. Biomedica 2014, 34(Supl. 1). in press.

88. The Uniprot Consortium: Update on activities at the Universal Protein Resource (UniProt) in 2013. Nucleic Acids Res 2013, 41(Database issue):D43-D47.

89. Pruitt KD, Tatusova T, Brown GR, Maglott DR: NCBI Reference Sequences (RefSeq): current status, new features and genome annotation policy. Nucleic Acids Res 2012, 40(Database issue):D130-D135.

90. Altschul SF, Madden TL, Schaffer AA, Zhang J, Zhang Z, Miller W, Lipman DJ: Gapped BLAST and PSI-BLAST: a new generation of protein database search programs. Nucleic Acids Res 1997, 25(17):3389-3402.

91. Do CB, Mahabhashyam MS, Brudno M, Batzoglou S: ProbCons: Probabilistic consistency-based multiple sequence alignment. Genome Res 2005, 15(2):330-340

92. Eddy SR: Profile hidden Markov models. Bioinformatics 1998, 14(9):755-763.

93. Letunic I, Doerks T, Bork P: SMART 7: recent updates to the protein domain annotation resource. Nucleic Acids Res 2012, 40(Database issue):D302-D305.

94. Warnes G, Bolker B, Bonebakker L, Gentleman R, Liaw WHA, Lumley T, Maechler M, Magnusson A, Moeller S, Schwartz M, Venables B: gplots: Various $\mathrm{R}$ programming tools for plotting data. In The Comprehensive $R$ Archive Network. 2009.

95. Goldovsky L, Cases I, Enright AJ, Ouzounis CA: BioLayout (Java): versatile network visualisation of structural and functional relationships. Appl Bioinformatics 2005, 4(1):71-74.

96. Garcia-Vallve S, Palau J, Romeu A: Horizontal gene transfer in glycosyl hydrolases inferred from codon usage in Escherichia coli and Bacillus subtilis. Mol Biol Evol 1999, 16(9):1125-1134.

97. Abascal F, Zardoya R, Posada D: ProtTest: selection of best-fit models of protein evolution. Bioinformatics 2005, 21(9):2104-2105.

98. Drummond A, Strimmer K: PAL: an object-oriented programming library for molecular evolution and phylogenetics. Bioinformatics 2001, 17(7):662-663.
99. Guindon S, Gascuel O: A simple, fast, and accurate algorithm to estimate large phylogenies by maximum likelihood. Syst Biol 2003, 52(5):696-704.

100. Notredame C, Higgins DG, Heringa J: T-Coffee: a novel method for fast and accurate multiple sequence alignment. J Mol Biol 2000, 302(1):205-217.

101. Di Tommaso P, Moretti S, Xenarios I, Orobitg M, Montanyola A, Chang JM, Taly JF, Notredame C: T-Coffee: a web server for the multiple sequence alignment of protein and RNA sequences using structural information and homology extension. Nucleic Acids Res 2011, 39(Web Server issue):W13-W17.

102. Wickham H: ggplot2: elegant graphics for data analysis. New York: Springer; 2009 .

103. Edgar RC: MUSCLE: a multiple sequence alignment method with reduced time and space complexity. BMC Bioinformatics 2004, 5:113.

104. Edgar RC: MUSCLE: multiple sequence alignment with high accuracy and high throughput. Nucleic Acids Res 2004, 32(5):1792-1797.

105. Korber B: HIV Signature and Sequence Variation Analysis. In Computational Analysis of HIV Molecular Sequences. Edited by Rodrigo AG, Learn GH. Dordrecht, Netherlands: Kluwer Academic Publishers; 2000:55-72.

106. Nei M, Gojobori T: Simple methods for estimating the numbers of synonymous and nonsynonymous nucleotide substitutions. Mol Biol Evol 1986, 3(5):418-426.

doi:10.1186/1756-0500-7-440

Cite this article as: Mosquera-Rendón et al:: Evolutionary and sequencebased relationships in bacterial AdoMet-dependent non-coding RNA methyltransferases. BMC Research Notes 2014 7:440.

\section{Submit your next manuscript to BioMed Central and take full advantage of:}

- Convenient online submission

- Thorough peer review

- No space constraints or color figure charges

- Immediate publication on acceptance

- Inclusion in PubMed, CAS, Scopus and Google Scholar

- Research which is freely available for redistribution
C) Biomed Central 